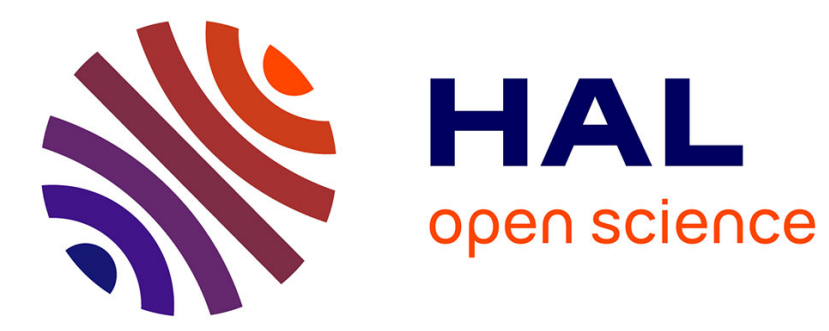

\title{
Du droit du patient de recevoir des soins antalgiques à l'obligation du médecin de prendre en charge la douleur
}

Clément Cousin

\section{To cite this version:}

Clément Cousin. Du droit du patient de recevoir des soins antalgiques à l'obligation du médecin de prendre en charge la douleur. Médecine et Droit, 2012, 116, pp.158-160. 10.1016/j.meddro.2012.05.001 . halshs-00757850

\section{HAL Id: halshs-00757850 \\ https://shs.hal.science/halshs-00757850}

Submitted on 12 Jan 2017

HAL is a multi-disciplinary open access archive for the deposit and dissemination of scientific research documents, whether they are published or not. The documents may come from teaching and research institutions in France or abroad, or from public or private research centers.
L'archive ouverte pluridisciplinaire $\mathbf{H A L}$, est destinée au dépôt et à la diffusion de documents scientifiques de niveau recherche, publiés ou non, émanant des établissements d'enseignement et de recherche français ou étrangers, des laboratoires publics ou privés. 


\title{
Du droit du patient de recevoir des soins antalgiques à l'obligation du médecin de prendre en charge la douleur.
}

\author{
Clément COUSIN \\ * * * \\ $*$
}

Résumé :

D'une obligation faite aux médecins et aux établissements de santé, le soulagement de la douleur est devenu un droit du patient avec la loi du 4 mars 2002. C'est un droit subjectif de la personnalité qui impose au médecin les obligations de résultat de prévention, d'évaluation, de prise en compte de la douleur et l'obligation de moyen de traitement de la douleur. Cette dernière obligation est tempérée par la liberté de prescription.

Pour assurer une sécurité juridique aux praticiens, il est impératif que ceux-ci renseignent scrupuleusement le dossier médical. Sont aussi très utiles les protocoles relatifs la douleur. Mais ceux-ci ne dispensent pas d'adapter la prise en charge au patient. En somme, la prise en compte de la douleur par le médecin est une obligation humaniste.

Mots clés :

Douleur ; Responsabilité médicale ; Droits des patients ; Dossier médical ; Protocole médical

From the patient's right to obtain analgesic treatment

Abstract:

to the obligation for the practitioner to take over pain

From a practitioner and hospital obligation, pain releasing has become a patient right. It is a subjective right of the personality imposing on the practitioner preventing, evaluating and accounting pain obligations of result and treating obligation of means. This obligation is tempered by the physician freedom to prescribe. In order to guarantee legal certainty for the practitioner, it is recommended to fill in carefully the patient's medical record and to remember that the medical protocol for pain releasing does neither ensure personal fitted care for the patient nor a complete pain consideration which is a humanist obligation.

$\underline{\text { Keywords : }}$

Pain; Medical responsibility; Patient rights; Medical record; Medical protocol

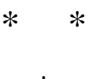

Doctorant en droit privé à l'IODE, UMR CNRS 6262, Rennes, France. Membre du réseau doctoral de l’École des Hautes Études en Santé Publique (EHESP).

Correspondance : clement.cousin@gmail.com 
Le droit pour le patient de recevoir des soins pour soulager sa douleur est vu comme « très » ou «plutôt important $»$ par les français ${ }^{1}$.

Ce droit est le fruit d'un mouvement récent. Avec le code de déontologie médicale de 1979 naît l'obligation pour le médecin de soulager la douleur. L'article $37 \mathrm{du}$ code en vigueur dispose que «le médecin doit s'efforcer de soulager les souffrances du malade par des moyens appropriés à son état et l'assister moralement. ${ }^{2}{ }^{\prime}$ Cette obligation a ensuite été imposée aux établissements de santé avec la loi du 4 février 1995. L'article L. 1112-4 du Code de la santé publique les oblige à mettre en œuvre des moyens pour prendre en charge la douleur du patient. Ces dispositions sont complétées par la charte du patient hospitalisé ${ }^{3}$, qui rappelle cet article. Le processus de certification des établissements de santé sanctionne la qualité des soins et consacre à la prise en charge de la douleur un critère de certification important ${ }^{4}$.

La loi du 4 mars 2002 crée l'article L. 1110-5 du Code de la santé publique. Son alinéa 4 dispose que «toute personne a le droit de recevoir des soins visant à soulager sa douleur. Celle-ci doit être en toute circonstance prévenue, évaluée, prise en compte et traitée. » Naît un droit du patient. C'est un droit subjectif en ce qu'il « existe sur la tête d'une personne ${ }^{5}$ », le patient. C'est un droit de la personnalité ; il « appartient essentiellement à une personne, ce qui fait qu'elle est elle et non pas une autre. ${ }^{6}{ }^{\prime}$

Ce droit subjectif de la personnalité s'adresse au médecin. Ainsi, il lui impose certaines obligations (I). La douleur étant in corpore, se pose la question de l'exercice de ce droit via la responsabilité et notamment la question de la preuve de la prise en charge de la douleur (II). Il existe enfin des situations critiques pour lesquelles le législateur a adapté ce droit commun de la prise en charge de la douleur (III).

I_Les obligations du médecin nées du droit du patient à bénéficier d'un soin antalgique

L'article L. 1110-5 alinéa 4 du Code de santé publique dispose in fine que la douleur «doit être en toute circonstance prévenue, évaluée, prise en compte et traitée. » Contrairement au traitement de la douleur qui est une obligation de moyen (B), les obligations de prévention, d'évaluation et de prise en compte sont des obligations de résultat $(\mathrm{A})$.

\section{A_L'obligation de résultat du médecin de prévenir, évaluer et prendre en compte la douleur}

La prévention consiste en la mise en place de mécanismes facilitant la prise en charge de la douleur (protocoles, comités de lutte contre la douleur, formation des soignants, etc.).

L'évaluation de la douleur peut être menée de deux manières. D'une part, en collaboration avec le patient par une auto-évaluation. Cela peut se faire via une échelle unidimentionnelle ${ }^{7}$ (la douleur n'est évaluée que selon l'intensité douloureuse) ou multidimentionnelle ${ }^{8}$ (la douleur est évaluée selon différentes composantes sensorielles et émotionnelles). D'autre part, la douleur peut être évaluée par des échelles comportementales ${ }^{9}$. Cette méthode est destinée aux patients communicants difficilement (enfants, personnes âgées, etc.). Le médecin ou l'infirmier renseigne alors lui-même le questionnaire et peut alors se faire une idée de la douleur du patient. 
L'obligation de prise en compte de la douleur est un impératif humaniste. D'une part elle impose une «écoute psychoaffective ${ }^{10}$ » de la part des soignants, les menant à prendre en considération la douleur du patient. D'autre part, cette obligation impose que la douleur soit considérée dans le parcours de soins. La douleur doit alors être un argument pour adapter ou renoncer à un acte médical, à un transport, ou, tout simplement, à l'aider dans les gestes quotidiens.

Ces obligations du médecin consistent en la réalisation d'un objet précis : il s'agit d'organiser la prévention ou l'évaluation. Concernant la prise en compte, il s'agit d'exiger un comportement humaniste ; l'objet de cette obligation est plus flou. Mais ces droits font naître des obligations de résultat, car ils ne sont pas fonction des aléas du corps humain. Il y a donc une obligation de résultat de prévention, d'évaluation et de prise en compte de la douleur.

\section{B_L'obligation du médecin de traiter la douleur tempérée par l'art médical}

Le traitement de la douleur passe par la prescription de soins. Le plus souvent il s'agit de traitements médicamenteux, mais de plus en plus sont prescrites des « méthodes non pharmacologiques ${ }^{11} »$.

L'acte de prescription est un acte médical. Il est admis que ces actes sont soumis à une obligation de moyen $^{12}$. L'application aux soins antalgiques de ce régime est logique : certaines douleurs sont insoignables (notamment certaines douleurs cancéreuses ou neuropathiques) et le médecin bénéficie, dans l'exercice de son art, d'une liberté de prescription.

Cette liberté de prescription est un principe cardinal de l'exercice médical ${ }^{13}$. Elle est cependant limitée par le respect des règles de l'art et par le respect de la volonté du patient (via notamment le devoir d'information). Cependant, le patient a un très faible pouvoir de contrainte, le médecin disposant d'une forte marge d'appréciation de l'opportunité de pratiquer un acte médical, qui est d'autant plus nécessaire que la contrepartie de cette liberté est l'engagement de sa responsabilité.

II_L_exercice du droit du patient à bénéficier d'un traitement antalgique : la recherche d'une sécurité juridique pour les soignants

L'exercice du droit du patient à bénéficier d'un traitement antalgique passe par l'engagement a posteriori de la responsabilité du médecin. La douleur est une sensation. Logiquement, vient la question de la preuve de la faute dans la prise en charge de la douleur par le médecin. En pratique, deux documents sont importants : le dossier médical (A) et les protocoles de prise en charge de la douleur (B).

\section{A_Le dossier médical : une preuve préconstituée}

L'évaluation de la douleur est, dans la plus part des cas, une auto-évaluation par le patient. Chaque patient est, en effet, son propre référentiel, il ne faut pas se fonder sur un niveau de douleur exprimé, mais sur une étude comparée de l'évolution et de l'auto-évaluation de la douleur par le patient et du traitement médical de la douleur. La preuve de la prise en charge est donc une étude comparative menée par un expert qui conclura à la faute si le traitement est aberrant vis-à-vis de l'évolution de la douleur ressentie.

Le document sur lequel se fondera l'expert est le dossier médical ${ }^{14}$, et plus spécifiquement le dossier infirmier qui contient notamment les transmissions infirmières écrites ${ }^{15}$. En somme, il s'agit de trouver la 
trace des évaluations de la douleur et des actes médicaux pratiqués. La preuve écrite est une condition importante de la sécurité juridique : les soignants doivent y veiller.

\section{$\underline{B}$ Le respect d'un protocole de prise en charge de la douleur : une sécurité juridique relative}

Il est certain que les protocoles de prise en charge sont utiles. Permettant une délégation d'actes aux infirmiers dans la prise en charge de la douleur ${ }^{16}$, ils sont ressentis comme juridiquement sécurisants. Produits de l'état de l'art médical, ils sont une réponse standardisée à des situations fréquentes. À ce titre, ils assurent un traitement effectif à la plupart des douleurs.

Cependant, ils ne sont pas l'assurance d'une protection légale parfaite.

D'une part, le protocole, conçu in abstracto, doit être adapté au patient. Le médecin doit délivrer les soins personnellement, en considération de la personne ${ }^{17}$. Les protocoles, conçus pour certaines prises en charge, ne prennent pas en compte les situations particulières.

D'autre part le protocole contribue à l'exécution des obligations de prévention, d'évaluation et de traitement de la douleur. Mais il omet de satisfaire l'obligation de prise en compte de la douleur. Au cours de litiges nombre de patients évoquent un défaut de communication, de considération de la part du médecin. Ces litiges se résolvent très souvent au stade de la médiation hospitalière ${ }^{18}$. Preuve en est que le protocole n'est pas une assurance tous risques.

La douleur est personnelle, elle appelle un traitement personnel.

III_Le droit spécial de la douleur : prendre en charge la douleur dans les situations d'exception

Cependant, dans certaines situations, le droit commun que nous avons visé est complété et adapté.

\section{A_Le traitement antalgique ayant pour effet secondaire de provoquer la mort}

L'article L. 1110-5 du Code la santé publique autorise le médecin à prescrire un traitement ayant pour but de soulager la souffrance, mais ayant comme effet secondaire d'abréger la vie. Cette prescription est soumise à la condition clinique que le patient soit dans une "phase avancée ou terminale d'une affection grave ou incurable » et à une obligation d'information étoffée : le médecin doit informer le malade, la personne de confiance et la famille. L'exigence d'inscription de ce traitement au dossier médical est inscrite dans la loi.

C'est donc le droit commun de la prise en charge de la douleur qui est ici complété.

\section{$\underline{B}$ La sédation consécutive à l'arrêt de traitement}

Enfin, le droit spécial de la douleur se tourne vers l'arrêt de traitements. L'arrêt de traitement est décidé dans certaines circonstances ${ }^{19}$ suivant une procédure collégiale. Dans ce cas, "le médecin, même si la souffrance du patient ne peut pas être évaluée du fait de son état cérébral, met en cuvre les traitements, notamment antalgiques et sédatifs, permettant d'accompagner la personne. ${ }^{20}{ }$ '

Alors disparait l'obligation d'évaluation, celle-ci étant impossible : le médecin prend alors en charge des douleurs inexprimables. 
${ }^{1}$ Les français et les droits des patients, Sondage réalisé par l'institut BVA pour le ministère chargé de la santé, Septembre 2010.

${ }^{2}$ Code de déontologie médicale issu du Décret n²006-120 du 6 février 2006, codifié à l'article R. 4127-1 ss. du Code de la santé publique.

${ }^{3}$ Issue de la circulaire $n^{\circ}$ DHOS/E1/DGS/SD1B/SD1C/SD4A/2006/90 du 2 mars 2006 relative aux droits des personnes hospitalisées et comportant une charte de la personne hospitalisée. La première charte est issue de la circulaire du 10 septembre 1974, circulaire prise en application du décret du 14 janvier 1974, nº74-27.

${ }^{4}$ Manuel de certification, HAS, Direction de l'amélioration de la qualité et de la sécurité des soins, Juin 2009, dite «Version 2010 ». Le critère 12.a est intitulé «Prise en charge de la douleur. »

${ }^{5}$ G. CORNU et Collectif, Vocabulaire juridique : Association Henri Capitant, 7e éd., Presses Universitaires de France - PUF, 2005, s.v. Subjectif.

${ }^{6}$ P. MALAURIE, Les personnes. Les incapacités, 2007, Defrénois, n²80.

${ }^{7}$ L'échelle visuelle analogique est la plus courante : sur une réglette sur laquelle le patient situe sa douleur, le soignant la note sous forme numérique, sur 10.

${ }^{8}$ Exemple : le questionnaire douleur de Saint-Antoine

${ }^{9}$ Exemple : échelle EDIN pour les nourrissons CEHOPS pour les enfants ou échelle Doloplus pour les personnes âgées.

${ }^{10}$ Selon les mots de J.-M. MANTZ, P. GRANDMOTTET, P. QUENEAU, Éthique et thérapeutique, Presses universitaires de Strasbourg, 1998.

${ }^{11}$ Selon le souhait du plan d'amélioration de la prise en charge de la douleur 2006-2010 (priorité n³).

${ }^{12}$ Voir not. Cass. 1re civ., 4 janv. 2005, nº3-13.579, JCP G. $n^{\circ}$ 20, 18 Mai 2005, II 10061, comm. P. MISTRETTA.

${ }^{13}$ Cf. art. L. 162-2 du Code de la sécurité sociale et l'art. R. 4327-8 du Code de la santé publique (art. 8 du Code de déontologie médicale).

${ }^{14}$ Cf. la composition du dossier médical visé à l'article R. 1112-2 du Code de la santé publique

${ }^{15}$ Les transmissions infirmières sont des notes prises par les infirmiers en vue de la communication entre les équipes et pour permettre une bonne continuité des soins. La transmission écrite décrit la réponse à une solution problématique (la cible), que cela passe par des traitements ou par l'information du médecin.

${ }^{16}$ Article R. 4311-8 du Code de santé publique, codifié par le décret nº 2004-802 du 29 juillet 2004.

${ }^{17} \mathrm{Cf}$. article $37 \mathrm{du}$ code de déontologie médicale.

${ }^{18}$ Voir à ce propos R. DUCLOS et C.DUQUESNE, « Protocoles contre la douleur : la loi nous aide-t-elle ? », Médecine \& Droit, vol. 2010, 100-101, janvier, p.p. 62-66.

${ }^{19}$ Voir sur ce point les articles L. 1110-5 al. 2, L. 1111-13 et L. 1111-4 du Code de la santé publique

${ }^{20}$ Art. 37 III. du Code de déontologie médicale, codifié à l'art. R. 4127-37 du Code de la santé publique modifié par le Décret n²012-107 du 29 janvier 2010 